Canad. Math. Bull. Vol. 19 (3), 1976

\title{
FULL IDEALS AND RING GROUPS IN $Z_{n}[x]$
}

\author{
BY
}

JOHN A. SUVAK

Introduction. If we add the operation of composition to the polynomial ring $R[x]$, where $R$ is a commutative ring with identity, we get a tri-operational algebra $\mathscr{A}=(R[x],+, \cdot, \circ)$. A full ideal or tri-operational ideal of $\mathscr{A}$ is the kernel of a tri-operational homomorphism on $\mathscr{A}$. This is equivalent $[4, \mathrm{pp}$. 73-74] to the following: A full ideal of $\mathscr{A}$ is a ring ideal $A$ of $R[x]$ such that $f \circ g \in A$ for every $f \in A$ and $g \in R[x]$. For a full ideal $A$ of $R[x]$ we can form the tri-operational algebra $(R[x] / A,+, \cdot, \circ)$ where $(R[x] / A, \circ)$ forms a monoid called the ring semi-group of $R[x]$ over $A$, denoted by $H_{R}(A)$. The group of units of $H_{R}(A)$ is called the ring group of $R[x]$ over $A$, denoted by $G_{R}(A)$.

For any ideal $I$ of $R,(I)=I[x]$ and $\{I\}_{R}=\{f \in R[x]: f(a) \in I$ for every $a \in R\}$ are full ideals of $R[x]$. Dickson [2] characterized the full ideals $\{I\}_{z}$ where $Z$ is the ring of integers, and Nöbauer [7] developed a general theory of ring groups and used these results to describe the ring groups $G_{Z}((I))$ and $G_{Z}(\{I\})$. It is natural to ask whether these results can be extended to the rings $Z_{n}$ of integers modulo $n$. In this paper, then, we will first characterize the full ideals $\{I\}_{Z_{n}}$, which we will shorten to $\{I\}_{n}$, and secondly we will describe the ring groups $G_{Z_{n}}((I))$ and $G_{Z_{n}}(\{I\})$, which we will shorten to $G_{n}((I))$ and $G_{n}(\{I\})$, for every $n$ and every ideal $I$ of $Z_{n}$.

1. The full ideals $\{I\}_{n}$. Since $Z_{n}$ is a principal ideal ring and, in fact, every ideal $I$ of $Z_{n}$ has a unique generator which is a divisor of $n$, our first problem is reduced to describing the ideals

$$
\{\langle d\rangle\}_{n}=\left\{f \in Z_{n}[x]: f(a) \equiv 0 \bmod d \quad \text { for every } a \in Z_{n}\right\}
$$

for every $n$ and every $d$ that divides $n$. We begin by giving a characterization for the full ideals $\{0\}_{n}=\{\langle n\rangle\}_{n}$, where 0 is the zero (full) ideal of $Z_{n}[x]$, and then we will see how these results can be used to find $\{I\}_{n}$ for any ideal $I$.

In characterizing $\{0\}_{n}$ for arbitrary $n$ we will make considerable use of results obtained by Dickson [2] for $Z[x]$. We thus consider the homomorphism $\phi: Z \rightarrow Z_{n}$ and the injective map $\psi: Z_{n} \rightarrow Z$, where $\phi$ is reduction modulo $n$, and the induced tri-operational homomorphism $\bar{\phi}: Z[x] \rightarrow Z_{n}[x]$ and injective map $\bar{\psi}: Z_{n}[x] \rightarrow Z[x]$, with $\phi \circ \psi$ and $\bar{\phi} \circ \bar{\psi}$ the identity maps on $Z_{n}$ and $Z_{n}[x]$ respectively. In Chapter II of [2] Dickson gives a method for constructing a

Received by the editors August 26, 1975. 
generating set for all residual polynomials modulo $n$; that is, all polynomials $f \in Z[x]$ with $f(a) \equiv 0 \bmod n$ for every $a \in Z$. The following lemma and theorem allow us to apply these results to solving our problem.

LEMMA 1. (1) If $f \in Z[x]$ and $a \in Z$, then $\phi(f(a))=\bar{\phi}(f)(\phi(a))$.

(2) If $f \in Z_{n}[x]$ and $a \in Z_{n}$, then $\psi(f(a)) \equiv \bar{\psi}(f)(\psi(a)) \bmod n$.

\section{Proof.}

(1) If $f=\sum c_{i} x^{i} \in Z[x]$ and $a \in Z$, then $\phi(f(a))=\phi\left(\sum c_{i} a^{i}\right)=\sum \phi\left(c_{i}\right)[\phi(a)]^{i}=$ $\bar{\phi}(f)(\phi(a))$.

(2) For $f \in Z_{n}[x]$ and $a \in Z_{n}$ we have, using (1) and the fact that $\phi \circ \psi$ and $\bar{\phi} \circ \bar{\psi}$ are identity maps, that $\phi(\psi(f(a)))=f(a)$ and also $\phi(\bar{\psi}(f)(\psi(a)))=$ $\bar{\phi}(\bar{\psi}(f))(\phi(\psi(a)))=f(a)$. Thus $\psi(f(a)) \equiv \bar{\psi}(f)(\psi(a)) \bmod n$.

Theorem 2. Let $\bar{\phi}$ and $\bar{\psi}$ be defined as above. Then,

(1) If $f$ is a residual polynomial modulo $n$, then $\bar{\phi}(f) \in\{0\}_{n}$.

(2) If $f \in\{0\}_{n}$, then $\bar{\psi}(f)$ is a residual polynomial modulo $n$.

\section{Proof.}

(1) If $f$ is a residual polynomial modulo $n$ and $a \in Z$, then $f(a) \equiv 0 \bmod n$ and $\phi(f(a))=0$. So $\bar{\phi}(f)(\phi(a))=0$ by Lemma 1 , and since $\phi$ is surjective we have $\bar{\phi}(f)(b)=0$ for every $b \in Z_{n}$ and thus $\bar{\phi}(f) \in\{0\}_{n}$.

(2) Let $f \in\{0\}_{n}$ and $a \in Z$. Then $\psi(\phi(a)) \equiv a \bmod n$. So by Lemma 1 , $\bar{\psi}(f)(a) \equiv \bar{\psi}(f)(\psi(\phi(a))) \equiv \psi(f(\phi(a))) \bmod n$. But $f(\phi(a))=0$ since $f \in\{0\}_{n}$. Thus $\bar{\psi}(f)(a) \equiv 0 \bmod n$.

This theorem together with results of Dickson [2] in characterizing residual polynomials can be applied in a simple way to our case to get the following results describing $\{0\}_{n}$. If $p$ is a prime and $t \leq p$, then Theorem 27 of [2] gives,

$$
\{0\}_{p^{t}}=\left\langle p^{t-1}\left(x^{p}-x\right), p^{t-2}\left(x^{p}-x\right)^{2}, \ldots, p\left(x^{p}-x\right)^{t-1},\left(x^{p}-x\right)^{t}\right\rangle .
$$

Note that taking $t=1$ we get the principal ideal $\{0\}_{p}=\left\langle x^{p}-x\right\rangle$. If we now define a map $\pi: Z^{+} \rightarrow Z[x]$ by $\pi(k)=x(x-1)(x-2) \cdots(x-k+1)$ and identify $\bar{\phi}(\pi(k))$ with $\pi(k)$, then Theorem 2 above and equation (32) of [2] give that each $f \in\{0\}_{n}$ of degree $m$ can be expressed in the form $f=$ $a_{2} \pi(2)+a_{3} \pi(3)+\cdots+a_{m} \pi(m)$ where $k ! a_{k}=0$ for $k=2,3, \ldots, m$. Moreover we are able to give a method for constructing a generating set for $\{0\}_{n}$. For $m$ a positive integer let $\mu(m)$ denote the least positive integer such that $\mu(m)$ ! is divisible by $m$. Now for a given $n$, partition the divisors $d>1$ of $n$ into sets by the equivalence relation that identifies divisors with the same $\mu$ value. Choose as a representative of each class the largest $d$ of that class and let $d_{1}, d_{2}, \ldots, d_{\text {s }}$ denote these representatives. Then Theorem 2 together with equation (27) and Theorem 28 of [2] give us, 
COROLlary 3. Let $n$ be arbitrary and $d_{1}, d_{2}, \ldots, d_{s}$ be the divisors of $n$ selected above. Then

$$
\{0\}_{n}=\left\langle\frac{n}{d_{1}} \pi\left(\mu\left(d_{1}\right)\right), \frac{n}{d_{2}} \pi\left(\mu\left(d_{2}\right)\right), \ldots, \frac{n}{d_{s}} \pi\left(\mu\left(d_{s}\right)\right)\right\rangle .
$$

As an example the reader can verify that $\{0\}_{30}=\langle 15 \pi(2), 5 \pi(3), \pi(5)\rangle$.

We now use the above results to characterize the full ideals $\{I\}_{n}$ for arbitrary $n$ and any ideal $I$ of $Z_{n}$. Let $\{I\}_{n}=\{\langle d\rangle\}_{n}$ where $d$ divides $n$. Then the maps $\alpha: Z_{n} \rightarrow Z_{d}$ and $\bar{\alpha}: Z_{n}[x] \rightarrow Z_{d}[x]$, which are reduction modulo $n$, are ring epimorphisms, and, as in Lemma 1 , for any $f \in Z_{n}[x]$ and $a \in Z_{n}$ we have $\alpha(f(a))=\bar{\alpha}(f)(\alpha(a))$. We also have the obvious injection maps $\beta: Z_{d} \rightarrow Z_{n}$ and $\bar{\beta}: Z_{d}[x] \rightarrow Z_{n}[x]$ and these are such that $\alpha \circ \beta$ and $\bar{\alpha} \circ \bar{\beta}$ are identity maps. We first show,

LEMMA 4. Let $\bar{\alpha}$ be as given above and let $f \in Z_{n}[x]$. Then $f \in\{\langle d\rangle\}_{n}$ if and only if $\bar{\alpha}(f) \in\{0\}_{d}$.

Proof. If $f \in\{\langle d\rangle\}_{n}$, then $f(a) \equiv 0 \bmod d$ for every $a \in Z_{n}$ and hence $\alpha(f(a))=$ $\bar{\alpha}(f)(\alpha(a))=0$. Since $\alpha$ is surjective we have $\bar{\alpha}(f)(b)=0$ for tvery $b \in Z_{d}$ and $\bar{\alpha}(f) \in\{0\}_{d}$. Conversely, if $\bar{\alpha}(f) \in\{0\}_{d}$, then for any $a \in Z_{n}$ we have $0=$ $\bar{\alpha}(f)(\alpha(a))=\alpha(f(a))$. So $f(a) \equiv 0 \bmod d$ and $f \in\{\langle d\rangle\}_{n}$.

With this we can prove our desired result.

THEOREM. If $\{0\}_{d}=\left\langle f_{1}, f_{2}, \ldots, f_{k}\right\rangle$ and $d$ divides $n$, then $\{\langle d\rangle\}_{n}=$ $\left\langle\bar{\beta}\left(f_{1}\right), \bar{\beta}\left(f_{2}\right), \ldots, \bar{\beta}\left(f_{k}\right), d\right\rangle$ where $d$ is the constant polynomial $f=d$ in $Z_{n}[x]$.

Proof. Suppose $f \in\left\langle\bar{\beta}\left(f_{1}\right), \bar{\beta}\left(f_{2}\right), \ldots, \bar{\beta}\left(f_{k}\right), d\right\rangle$, so $f=d g+\sum_{i=1}^{k} \bar{\beta}\left(f_{i}\right) g_{i}$ with $g$, $g_{i} \in Z_{n}[x], i=1,2, \ldots, k$. Since $\bar{\alpha}$ is a ring homomorphism and $\bar{\alpha} \circ \bar{\beta}$ is the identity map on $Z_{d}[x]$ we get $\bar{\alpha}(f)=\sum_{i=1}^{k} f_{i} \bar{\alpha}\left(g_{i}\right) \in\{0\}_{d}$, and so by Lemma 4 we have $f \in\{\langle d\rangle\}_{n}$. Conversely, suppose $f \in\{\langle d\rangle\}_{n}$. Then $\bar{\alpha}(f) \in\{0\}_{d}$ by Lemma 4 and we can write $\bar{\alpha}(f)=\sum_{i=1}^{k} f_{i} g_{i}$ for some $g_{i} \in Z_{d}[x]$. Now consider the polynomial $h=\sum_{i=1}^{k} \bar{\beta}\left(f_{i}\right) \bar{\beta}\left(g_{i}\right)$. Then $\bar{\alpha}(h)=\sum_{i=1}^{k} f_{i} g_{i}$ and $\bar{\alpha}(f)=\bar{\alpha}(h)$ or $\bar{\alpha}(f-h)=0$. Hence $f-h=d g$ for some $g \in Z_{n}[x]$ and so

$$
f=d g+h=d g+\sum_{i=1}^{k} \bar{\beta}\left(f_{i}\right) \bar{\beta}\left(g_{i}\right)
$$

and $f \in\left\langle\bar{\beta}\left(f_{1}\right), \bar{\beta}\left(f_{2}\right), \ldots, \bar{\beta}\left(f_{k}\right), d\right\rangle$ and the theorem is proved.

Thus we can obtain a set of generators for $\{\langle d\rangle\}_{n}$ by sort of lifting the generators of $\{0\}_{d}$, which we can construct from Corollary 3 , to $Z_{n}[x]$ and throwing in the constant polynomial $f=d$.

2. The ring groups $G_{n}((I))$. Nöbauer [7] obtained results concerning the ring groups $G_{z}((I))$. The following theorem and corollary allow us to apply these results to our problem. 
THEOREM 6. Let $I=\langle n\rangle, n$ a positive integer, be an ideal of $Z$. Then $G_{Z}((I)) \simeq$ $G_{n}((0))$.

Proof. Since $H_{n}((0))$ is the semi-group $\left(Z_{n}[x]\right.$, ०), we first define $\theta: H_{Z}((I)) \rightarrow$ $H_{n}((0))$ by $\theta(f+(I))=\bar{\phi}(f)$. Now $\theta$ is well-defined, for if $f+(I)=g+(I)$ then $f-g \in(I)$ and $\bar{\phi}(f-g)=0$, and so $\bar{\phi}(f)=\bar{\phi}(g)$. Clearly $\theta$ is an epimorphism since $\bar{\phi}$ is a tri-operational epimorphism. Also $\bar{\phi}(f)=\bar{\phi}(g)$ implies $\bar{\phi}(f-g)=0$ and $f-g \in(I)$ and so $\theta$ is a semi-group isomorphism. Since $\theta$ takes the identity of $H_{Z}((I))$ onto the identity of $H_{n}((0))$, the restriction of $\theta$ to $G_{Z}((I))$ is an isomorphism onto $G_{n}((0))$.

It immediately follows by factoring that,

COROllary 7. Let $I=\langle m\rangle$ be an ideal of $Z_{n}$ where $m$ divides $n$. Then $G_{n}((I)) \cong G_{m}((0))$.

Thus we see that we will have the structure of $G_{n}((I))$ for every $n$ and every ideal $I$ of $Z_{n}$ if we can only obtain the structure of $G_{n}((0))$ for every $n$.

The case of $n=p$, a prime, is particularly interesting since it can be generalized to obtaining the structure of $G_{D}((0))$, the group of units of $D[x]$ under composition, for any integral domain $D$. Let $D^{+}$be the additive group of $D$ and $U(D)$ be the group of multiplicative units of $D$. It is well known that

$$
G_{D}((0))=\{a+b x \in D[x]: b \in U(D)\} .
$$

We can now express $G_{D}((0))$ as a semi-direct product. If we let

$$
B=\{a+x: a \in D\} \text { and } H=\{b x: b \in U(D)\},
$$

then $B$ is a normal subgroup of $G_{d}((0)), H$ is a subgroup of $G_{D}((0))$, $B \cap H=\{x\}$, and $\left.B H=G_{D}(q 0)\right)$. Thus $G_{D}((0))$ is a semi-direct product of $B$ and $H$, and in fact

$$
G_{D}((0)) \simeq B X_{\theta} H
$$

where $\theta: H \rightarrow \operatorname{Aut}(B)$ is given by $\theta(b x)(a+x)=b a+x$. Of course $B \simeq D^{+}$and $H \simeq U(D)$ and we can also express

$$
G_{D}((0)) \simeq D^{+} X_{\theta} U(D)
$$

where $\theta: U(D) \rightarrow \operatorname{Aut}\left(D^{+}\right)$by $\theta(d)(a)=d a$.

Thus we have characterized $G_{D}((0))$ as a semi-direct product for any integral domain $D$. Returning to the case of $Z_{p}$, a field, we get: Let $p$ be a prime. Then $f \in Z_{p}[x]$ belongs to $G_{p}((0))$ if and only if $f$ has the form $f=a+b x, b \neq 0$, and

$$
G_{p}((0)) \simeq Z_{p}^{+} X_{\theta} Z_{p}^{*}
$$

where $Z_{p}^{*}$ is the multiplicative group of non-zero elements of $Z_{p}$ and $\theta: Z_{p}^{*} \rightarrow$ $\leftarrow \operatorname{Aut}\left(Z_{p}^{+}\right)$is given by $\theta(a)(c)=a c$ for $a \in Z_{p}^{*}$ and $c \in Z_{p}^{+}$. 
Observe that $\left|G_{p}((0))\right|=p(p-1)$, and since $Z_{p}^{+} \simeq \sigma(p)$ and $Z_{p}^{*} \simeq \sigma(p-1)$ we also have $G_{p}((0))$ expressed as a semi-direct product of cyclic groups. Furthermore, in this case we can identify these semi-direct products $G_{p}((0))$ as known groups. For any three positive integers $m, n$ and $k$ the metacyclic group $M(m, n, k)$ is defined [3, p. 462] as the group generated by two elements $a$ and $b$ satisfying $a^{m}=1, b^{n}=1$ and $b a b^{-1}=a^{k}$ where $k^{n} \equiv 1 \bmod m$. We then have,

Theorem 8. Let $p$ be a prime and $n$ a primitive root of $p$. Then $G_{p}((0)) \simeq$ $M(p, p-1, n)$.

Proof. If we let $f=1+x$ and $g=n x$, then $f, g \in G_{p}((0))$ with $f^{p}=x, g^{p-1}=x$ and $g \circ f \circ g^{-1}=n+x=f^{n}$ with $n^{p-1} \equiv 1 \bmod p$. Also it is easily seen that $f$ and $g$ generate $G_{p}((0))$ since $f^{\alpha} g^{\beta}=\alpha+n^{\beta} x$ and $n$ is a primitive root of $p$.

To describe $G_{n}((0))$ for composite $n$ we use Theorem 6 to apply results Nöbauer [7] obtained for the ring groups $G_{z}((I))$. If $n$ is a power of a prime then we can use equations (11) and (12) of [7] and Theorem 6 to determine which elements of $Z_{p^{\prime}}[x]$ belong to $G_{p^{\prime}}((0))$. Specifically, if $p$ is a prime and $t>0$, then $f \in G_{p^{\prime}}((0))$ if and only if it can be expressed in the form

$$
f=a+b x+p x^{2} \alpha(x)
$$

with $b \in U\left(Z_{p^{t}}\right)$, so $b \not \equiv 0 \bmod p$, and $\alpha(x) \in Z_{p^{\natural}}[x]$. Thus we have a structural representation for $G_{p^{t}}((0))$ to the extent that elements of $Z_{p^{t}}[x]$ are identifiable as elements of $G_{p^{\prime}}((0))$ or not.

Finally, for $n$ arbitrary, $n=p_{1}^{t_{1}} p_{2}^{t_{2}} \cdots p_{r}^{t_{r}}, p_{i} \neq p_{j}$ for $i \neq j$, we can use Theorem 6 to apply a result of Nöbauer [7, p. 257] to get

$$
G_{n}((0)) \simeq G_{p_{1}} t_{1}((0)) X G_{p_{2}} t_{2}((0)) X \cdots X G_{p_{r}} t_{r}((0)) .
$$

From this we get the result,

THEOREM 9. $G_{n}((0))$ is finite if and only if $n$ is square free.

Proof. If $n=p_{1} p_{2} \cdots p_{r}$ is square free, then $\left|G_{n}((0))\right|=\prod_{i=1}^{r} p_{i}\left(p_{i}-1\right)$ and hence is finite, and if $n$ is not square free then $G_{n}((0))$ is not finite since $G_{p^{i}}((0))$ is not finite for $i>1$.

3. The ring groups $G_{n}(\{I\})$. This problem is again reduced to describing $G_{n}(\{\langle d\rangle\})$ for every $n$ and every $d$ that divides $n$. The following theorem and corollary give us a further reduction and allow us to apply the results of Nöbauer $[5,6,7]$ to this case.

TheOREM 10. Let $I=\langle n\rangle, n$ a positive integer, be an ideal of $Z$. Then $G_{Z}(\{I\}) \simeq G_{n}(\{0\})$.

Proof. We first define $\theta: H_{Z}(\{I\}) \rightarrow H_{n}(\{0\})$ by $\theta(f+\{I\})=\bar{\phi}(f)+\{0\}_{n}$. Now $\theta$ is well-defined, for if $f+\{I\}=g+\{I\}$ then $f-g \in\{I\}$ and by Theorem 2 we have 
$\bar{\phi}(f-g)=\bar{\phi}(f)-\bar{\phi}(g) \in\{0\}_{n}$. Also $\theta$ is an epimorphism since $\phi$ is a trioperational epimorphism. Furthermore $\theta$ is injective, for if $\phi(f)+\{0\}_{n}=$ $\bar{\phi}(g)+\{0\}_{n}$ then $\bar{\phi}(f-g) \in\{0\}_{n}$ and $f-g \in\{I\}$, and so $f+\{I\}=g+\{I\}$. Thus $\theta$ is a semi-group isomorphism. Since $\theta$ takes the identity of $H_{Z}(\{I\})$ onto the identity of $H_{n}(\{0\})$, the restriction of $\theta$ to $G_{Z}(\{I\})$ is an isomorphism onto $G_{n}(\{0\})$.

Again by factoring we get,

COROllary 11. Let $I=\langle m\rangle$ be an ideal of $Z_{n}$ where $m$ divides $n$. Then $G_{n}(\{I\}) \simeq G_{m}(\{0\})$.

Thus we reduce the problem to that of finding $G_{n}(\{0\})$ for arbitrary $n$.

The case of $n=p$, a prime, is taken care of by a more general result. If $F=G F\left(p^{k}\right)$ is the field of $p^{k}$ elements, then using Satz 10 of [7] and Theorem 3 of [1] it is easy to see that

$$
G_{F}(\{0\}) \simeq S_{p^{k}}
$$

where $S_{p^{k}}$ is the symmetric group on $p^{k}$ elements. Taking $k=1$ gives

$$
G_{p}(\{0\}) \simeq S_{p}
$$

The case of $n$ composite can be solved by applying Theorem 10 to results of Nöbauer [5, 6]. First, we find the structure of $G_{p^{t}}(\{0\}), p$ a prime, $t>1$. From Satz IV of [6] and Theorem 10 we know that all the elements of $G_{p^{t-1}}(\{0\})$ which have a representative of the form

$$
f=a_{0}+a_{1} x+p a_{2} x^{2}+\cdots+p^{t-2} a_{t-1} x^{t-1}
$$

form a subgroup of $G_{p^{t-1}}(\{0\})$. If we denote this subgroup by $B_{p^{t-1}}$ then Satz V of [6] gives us that $G_{p^{\prime}}(\{0\})$ is isomorphic to the complete monomial group of degree $p$ of $B_{p^{t-1}}$. (See Ore [8] for a general study of monomial groups.) We can also obtain a formula for the order of $G_{p^{t}}(\{0\})$. Let $n=p^{t}, p$ a prime, be given. For a positive integer $i$ let $\varepsilon(i)$ denote the exponent of $p$ in the prime factorization of $i$, and let $s$ be the largest integer for which $s+\varepsilon(s)<t$ and let $T=\sum_{i=0}^{s}(t-i-\varepsilon(i))$. Then equation (15) of [6] gives us that

$$
\left|G_{p^{t}}(\{0\})\right|=p !(p-1)^{p} p^{(T-2) p}
$$

Finally we can obtain $G_{n}(\{0\})$ for arbitrary $n$. If $n=p_{1}^{t_{1}} p_{2}^{t_{2}} \cdots p_{r}^{t_{r}}$ is any positive integer, then applying Theorem 10 to a result of Nöbauer [7, p. 257] we get

$$
G_{n}(\{0\}) \simeq G_{p_{1}} t_{1}(\{0\}) X G_{p_{2}} t_{2}(\{0\}) X \cdots X G_{p_{r}} t_{r}(\{0\})
$$

Thus we can find $G_{n}(\{I\})$ for any $n$ and any ideal $I$ of $Z_{n}$. Also, $G_{n}(\{I\})$ is finite for every $n$ and $I$, and in fact we can obtain a form 1 la for its order. 


\section{REFERENCES}

1. L. Carlitz, A Note on Permutation Functions over a Finite Field, Duke Math. Journal, 29 (1962), 325-332.

2. Leonard Eugene Dickson, Introduction to the Theory of Numbers, Dover Publications, Inc., New York, 1929.

3. Saunders MacLane and Garrett Birkhoff, Algebra, The MacMillan Company, New York, 1967.

4. Murray Mannos, Ideals in Tri-operational Algebras I, Reports Math. Colloquium, 7 (1946), 73-79.

5. Wilfred Nöbauer, Über Gruppen von Restklassen nach Restpolynomidealen, Sitsber. Akad. Wiss. Wien, Abt. II, 162 (1953), 207-233.

6. Wilfred Nöbauer, Gruppen von Restpolynomidealrestklassen nach Primzahlpotenzen, Monatsh. f. Math., 59 (1955), 194-202.

7. Wilfred Nöbauer, Über die Operation des Einsetzen in Polynomringen, Math. Ann., 134 (1958), 248-259.

8. Oystein Ore, Theory of Monomial Groups, Trans. Amer. Math. Soc., 51 (1942), 15-64.

4 Outerbridge Street,

ST. JOHN's,

Newfoundland, Canada,

A1E 3X5 